\title{
El Protagonismo Femenino en la Radicalización de la Democracia Venezolana Bolivariana
}

\author{
The Feminine Protagonism in the Radicalization of Bolivarian Venezuelan Democracy
}

\author{
Rickard Lalander \\ Universidad de Helsinki \\ lalander@yahoo.com \\ Juan Velásquez-Atehortúa
Universidad de Estocolmo
juan.velasquez@socant.su.se
}

Resumen

El objetivo del presente artículo es examinar el creciente protagonismo de mujeres en las nuevas estructuras participativas dentro del esquema de transformación de la democracia venezolana en tiempos de la administración del Presidente Hugo Rafael Chávez Frías, particularmente a partir de 2006. $\mathrm{Si}$ bien es cierto que se ha acentuado el grado de personalismo político durante la era chavista, al mismo tiempo el sistema político ha cambiado de carácter, con más participación popular e inclusión social en las bases locales. Se enfatizarán los cambios en cuanto al surgimiento de mujeres en las estructuras políticas locales y su protagonismo y empoderamiento en la democracia participativa radical. Para visibilizar eso se analiza sobre todo un modelo participativo de la llamada Revolución Bolivariana: los Consejos Comunales, pero también habrá examinación de otros mecanismos y dimensiones de la participación popular. Dentro de estos esquemas una gran cantidad de mujeres de los sectores populares han encontrado su propio espacio de empoderamiento. Teóricamente, la investigación se vincula con los debates de democracia radical y participativa, relaciones Estado-ciudadanía y el empoderamiento de las mujeres que anteriormente estaban excluidas de las esferas públicas..

Palabras clave: Consejos Comunales; democracia radical; empoderamiento; lucha de género; Venezuela.

\begin{abstract}
The aim of this article is to examine the growing protagonism of women in the recently developed participatory structures within the framework of democratic transition in Venezuela, during the presidency of Hugo Rafael Chávez Frías, mainly from 2006 onwards. While the degree of personalized political symbolic power has deepened during the period, at the same time the political system has changed, towards a model characterized by broader political participation and social inclusion at grassroots level. The insurgence of popular sector women in the local political structures will be emphasized, as well as the female protagonism and empowerment in the radical participatory democracy in progress. In order to make this issue visible, the analytical focus will be placed mainly on one participatory model of the socalled Bolivarian Revolution: the Community Councils, although other relevant mechanisms and dimensions of popular participation will be dealt with. Through these frameworks, a growing number of women from the popular sectors have found their own space of empowerment. Theoretically, this research connects to radical and participatory democracy debates, changing State-Citizenship relations and the empowerment of women who were previously excluded from the public spheres.
\end{abstract}

Keywords: Community councils; empowerment; gender struggle; radical democracy; Venezuela. 


\section{Presentación}

\begin{abstract}
Mujeres, todas, justamente conocen la realidad, diagnostican, planifican, ejecutan. Son las que se relacionan, las que articulan. Una de las cosas que uno dice: '¿Bueno es que las mujeres tenemos mayores responsabilidades que los hombres?' Uno tiene que preguntarse eso. ¿Tenemos más sensibilidad social? ¿Humana? Porque la mayoría realmente somos mujeres. Las que estamos al frente de esos espacios. Que si hay una responsabilidad, bueno hay que cumplirla así llueva, truene y relampaguee, caiga un rayo, lo que sea...ahí en esos espacios... (Mata, entrevista, Macuto, 3 de junio, 2011).
\end{abstract}

En tiempos recientes, las mujeres han logrado importantes conquistas políticas y simbólicas, no sólo se han elegido presidentas mujeres de varias naciones latinoamericanas, sino igualmente la proporción de mujeres parlamentarias, alcaldesas, gobernadoras etcétera tiende a fortalecerse en diversas partes del continente. El presente estudio trata del empoderamiento de las mujeres de los sectores populares urbanos en Venezuela. Las mujeres venezolanas, principalmente de los sectores sociales tradicionalmente marginalizados, son protagonistas en nuevos modelos de participación política: por ejemplo en los Comités de barrios, Mesas Técnicas de Agua y de Tierra, Salas de Batalla Social, y, las estructuras participativas examinadas en el presente trabajo; los Consejos Comunales. El presente artículo explorará algunas particularidades de la transformación de la democracia venezolana en tiempos del gobierno de Hugo Rafael Chávez Frías, con un enfoque específico en el surgimiento de mujeres en las nuevas estructuras participativas desde 2006.

Innegablemente, el carácter de la democracia ha sido transformado desde 1999. Venezuela - hace años percibida como una excepción política en el continente, por su estabilidad democrática a través de dos partidos políticos ${ }^{1}$ entre 1958 y los años 90, mediante un modelo de democracia liberal representativa (y excluyente) - ahora se perfila como promotor de la izquierdización de América Latina. Es axiomático que el gobierno actual venezolano se ha caracterizado por un alto grado de personalismo, e inclusive un culto al máximo líder del proceso. Sin embargo, en términos de democratización al nivel local, asimismo se han desarrollado nuevos modelos caracterizados por creciente participación popular e inclusión social en las bases locales, es decir una radicalización de la democracia.

En este artículo se enfocarán los mecanismos para animar a la participación política vecinal e inclusión social. Especialmente se enfatizará los cambios en cuanto a la emergencia de mujeres en las estructuras políticas locales y su protagonismo en la democracia participativa. ¿Qué significa en términos de lucha por la igualdad de género, ciudadanía y cambios del espacio democrático? Para lograr visibilizar eso se decidió enfocar más que todo en uno de los modelos participativos de la llamada Revolución Bolivariana: los Consejos Comunales y su impacto desde 2006. Dentro de este esquema una gran cantidad de mujeres de los sectores populares han encontrado su propio lugar de empoderamiento. Teóricamente, el estudio se vincula con los debates de democracia radical y participativa, relaciones Estado-ciudadanía y el empoderamiento de las mujeres que anteriormente estaban excluidas de las esferas públicas. Con respecto a la selección de espacios institucionales para el enfoque analítico del estudio, esta se justifica considerando que los Consejos Comunales son estructuras fundamentales en el modelo de democracia participativa social en las cuales las mujeres especialmente en los barrios marginales - han ocupado importantes funciones y un evidente protagonismo.

Metodológicamente, el presente trabajo se apoya en entrevistas semi - estructuradas y observaciones participativas durante trabajo de campo en Venezuela, particularmente durante unos meses en 2010 y 2011 y en Caracas y los estados Vargas, Zulia y Apure. Es importante aclarar que el objetivo no es ofrecer los datos exactos de la proporción relativa de participación y protagonismo de las mujeres en las unidades organizativas estudiadas. El estudio tampoco intenta examinar la eficiencia de la labor hecha por ellas, sino la idea central es más bien explorar y analizar los avances de las mujeres en los Consejos Comunales y otras dimensiones participativas en el contexto de democratización y ciudadanía desde los ángulos de las mujeres y las organizaciones de base del modelo de poder popular en la Venezuela Bolivariana. Es importante problematizar los nuevos contextos democráticos a nivel local-vecinal. Por consiguiente, se analizarán los Consejos Comunales y la radicalización de la democracia venezolana, pero conectando estos procesos con las perspectivas de género dentro de las estructuras participativas.

En cuanto a la estructura y el contenido del artículo, luego de la presente contextualización introductoria habrá una reflexión sobre algunos obstáculos históricos y actuales para las expectativas de lograr un empoderamiento de las mujeres marginalizadas. En seguida se discute sobre la 
conceptualización de ciudadanía insurgente en el contexto del empoderamiento de las mujeres. A continuación habrá un resumen analítico de ciertas dimensiones del modelo político que se ha desarrollado en tiempos del mandato de Hugo Chávez. Después se presentan tres apartados centrales, que enfocan: los Consejos Comunales, la expansión de la democracia radical participativa y una recapitulación de las nuevas estructuras participativas y el protagonismo de las venezolanas que anteriormente estaban marginalizadas. El artículo se redondea con algunas conclusiones acertadas.

\section{Contextualización Histórica}

Hay diferentes desafíos y obstáculos de carácter socio - cultural al hablar de la lucha de igualdad de género en América Latina. Los valores culturales y tradiciones relacionados al machismo y el marianismo agrupan unos de los retos principales para avanzar en esta lucha, y son características típicas asimismo en la sociedad venezolana. El concepto del machismo expresa la idea que el varón sería superior a la hembra e incluye frecuentemente componentes de arrogancia y agresiones en cuanto a las relaciones hombre - mujer. La noción del marianismo deriva de la imagen de la Virgen María; es decir, la mujer debería ser virgen y negarse a si misma. Asimismo, la maternidad es un papel central en el marianismo, la mujer debe ser una 'buena madre'. Lógicamente esta ecuación de virgen y buena madre a la vez resulta imposible en la práctica. El machismo y el marianismo se reproducen en normas culturales, y contribuyen a la justificación en que el hombre puede tener su vida fuera de la casa, mientras que la 'buena' madre/esposa/mujer debe quedarse cuidando el hogar. Por ende, para las mujeres la maternidad es la función/responsabilidad principal, mientras que para los hombres la paternidad no es primordial. Por supuesto, las mujeres de todos los sectores sociales si pasan las esferas de lo privado y lo público, por ejemplo mediante el trabajo en actividades de la comunidad/vecindad. No obstante, frecuentemente se perciben estas actividades como secundarias o complementarias a lo que hacen normalmente (amas de casa). Estas distinciones contribuyen al fortalecimiento y mantenimiento de los estereotipos de machismo y marianismo (CRASKE, 1999).

\section{Empoderamiento de las Mujeres Marginalizadas: la Ciudadanía Insurgente}

Ahora bien, es primordial enfatizar que hay caminos para alejarse de estructuras sociales patriarcales y lograr avances en el empoderamiento individual y colectivo de las mujeres. Primero que todo se debe definir el concepto y luego problematizar (los diferentes caminos/procesos hacia) el empoderamiento:

(Empoderamiento es el) proceso a través del cual personas oprimidas puedan obtener algún control sobre sus vidas mediante el compartimiento con otros en el desarrollo de actividades y estructuras que permitan involucramiento aumentado a la gente en asuntos que les afectan directamente. En su curso, la gente vuelve habilitada de gobernarse a sí misma eficientemente (BYSTYDZIENSKI apud CRASKE, 1999, p. 23)

Es substancial plantearse los dilemas considerados más allá de las perspectivas euro-céntricas de empoderamiento. Por ejemplo, se puede partir desde el contexto de realidades socio-espaciales de los barrios y sectores populares latinoamericanos. En este contexto es pertinente subrayar la dimensión socio-económica desde el ángulo de las mujeres. En reportes de las Naciones Unidas desde el año 2000, se concluyó que la pobreza tiene cara de mujer. La gran parte de los 1.500 millones de personas que viven en condiciones de ingresos de un dólar o menos al día de hecho son mujeres. Aún más, se ha ampliado a nivel mundial la brecha que separa a los hombres de las mujeres aferrados en el ciclo de la pobreza ha seguido, un fenómeno que ha llegado a conocerse como 'la feminización de la pobreza'. En todo el mundo, las mujeres ganan como promedio un poco más del 50\% de lo que ganan los hombres (NACIONES UNIDAS, 2000). Tomando en consideración que la feminización de la pobreza es un fenómeno medible y absolutamente materializado en los barrios informales de las ciudades latinoamericanas la siguiente pregunta sería: ¿Qué hace el estado y qué hacen las mujeres para sobreponerse a esta situación? Para acercarnos a esta cuestión temática es oportuno reflexionar teóricamente sobre el empoderamiento de las mujeres y la conceptualización de ciudadanía. El antropólogo James Holston ha planteado el concepto de ciudadanía insurgente, la cuál muestra cómo de los barrios populares emergen formas organizativas que transforman el marco de derechos vigentes en las sociedades. A través de este concepto teórico, Holston critica interpretaciones anteriores en cuanto a las condiciones sociales en los sectores marginalizados y destaca la importancia de explorar la capacidad real de 
acción de los ciudadanos marginalizados y no reconocidos para superponerse a la pobreza y la marginalidad. Sustenta que las movilizaciones de los excluidos por ser incluidos en las tomas de decisiones, podría conllevar a que estos ciudadanos introduzcan invenciones significativas tanto al marco de derechos de ciudadanía como en los procesos de cimentación de sus barrios y de las ciudades mismas. Holston indica asimismo que las mujeres son los actores más presentes en estas movilizaciones por la ciudadanía insurgente, por lo que es importante aclarar el papel que han jugado con su movilización tanto en la democratización de la sociedad como en el desarrollo urbano-espacial (HOLSTON, 2009).

Tradicionalmente, el trabajo de las mujeres en la economía del cuidado de la vida se ha considerado como conectado al 'destino subordinado' de las mujeres como lo apuntábamos anteriormente en la argumentación sobre el marianismo. Este trabajo está también parcialmente ligado a la labor que realizan para construir y cuidar físicamente sus viviendas, sus barrios y de allí la ciudad 'informal'. Mediante este trabajo en la esfera del cuidado, las mujeres de los barrios establecen igualmente las bases a los valores inmateriales agregados que sus gestiones vecinales y urbanísticas generan en términos de culturas alternativas de complementariedad y solidaridad. Dentro de esta gestión vecinal urbana se encuentra del mismo modo la lucha por la dotación de servicios públicos domiciliarios con los que las mujeres, a la par que como lo hacen con las redes viales, también conectan los barrios marginales a los circuitos de bienestar y de sanidad del resto de la ciudad. Lo mismo podría señalarse en cuanto a las redes comerciales, en las que las mujeres juegan un papel preponderante para facilitar la vida cotidiana en sus barrios. Para todas estas gestiones los pobladoras de los barrios conciben formas organizativas y tecnologías propias que se constituyen en creaciones urbanas innovadoras y fundamentales para alcanzar las transformaciones y lograr la sostenibilidad de estructuras físicas como establecimientos sociales.

Sin embargo, no queremos expresar que todas éstas sean actividades exclusivamente de las mujeres amas de casa de los barrios. Solo queremos evidenciar que el 'destino' de estas mujeres, primariamente delimitado por las presunciones marianistas, circunscribe una identidad propia respecto a la flora de organizaciones de mujeres que hay en las ciudades, sean ellas feministas o no. Su labor 'informal' las distingue no sólo por los quehaceres invisibles a los ojos de la economía monetaria o no remunerada de la economía del cuidado, sino por la forma de construir la ciudad. Es esencialmente esta encrucijada de 'destinos' lo que hace que las amas de casa de los barrios populares se establezcan en alianzas de mujeres que se diferencian de otras mujeres por su manera de edificar la ciudad. Por lo demás, con su gestión urbana impulsan una estética alternativa que pone en entredicho los valores patriarcales dominantes, sean ellos socialistas o neoliberales.

Con respecto a lo que Holston llama el carácter insurgente de las movilizaciones de los habitantes de los barrios populares, como ya se enfatizó, quienes están más al frente de estas son las mujeres. Son ellas quienes principalmente se movilizan para construir nuevas instituciones didácticas que llevan a las mujeres a identificar los campos de batalla que las diferencia de otras organizaciones con un ideario feminista propio y autentico. A partir de este marco de 'hacer destino' son ellas quienes en gran medida se aparecen para liderar procesos de mejoramientos, de demandar servicios públicos domiciliarios, y con ellos de introducir nuevos modelos de derechos que van creando a la vez la (re)construcción de un estado de bienestar que desmiente la hegemonía patriarcal representada en la economía financiera, el trabajo formal asalariado y el manejo técnico de recursos naturales. Mediante estos combates socioinstitucionales las mujeres vienen construyendo instancias para el acceso universal al agua, a la energía básica, asimismo a la salud, la educación, la cultura, el esparcimiento público, y a la autonomía económica. Este carácter esencialmente insurgente se prolonga en los diagnósticos de la ciudad que las mujeres hacen para la construcción física de espacios e infraestructura de bienestar asentados en la complementariedad y la solidaridad colectiva. Este espejismo de las mujeres amas de casa barriales en este sentido las lleva a ser vigilantes para reclamar mejoramientos en los servicios ya existentes y asimismo para el suministro de los que aún no existen (VELÁSQUEZATEHORTÚA, 2011).

No obstante, aún sólo hay una limitada producción académica sobre diferentes formas de planificación insurgente $\mathrm{y} / \mathrm{o}$ popular, y menos aún con una alta participación femenina. Edward Soja, catedrático en planificación urbana y geografía política, acentúa la prevalencia del liderazgo de las mujeres como productoras y administradoras de las primeras ciudades que podría aproximarse a este modelo de ciudadanía insurgente de influencia femenina (SOJA, 2000). Hay una creciente cantidad de ciudades contemporáneas en las cuales se destaca el papel que empiezan a asumir las mujeres como constructoras no sólo de la ciudad sino también de la ciudadanía, desde sus barrios populares. Estos barrios populares se han convertido en espacios híbridos, que además de ser 
construidos en buena parte por las mujeres, son espacios donde coexisten prácticas de la ruralidad con lo urbano, lo moderno con lo antiguo, el consumismo con la pobreza extrema, lo formal y lo informal. De esta manera el barrio se erige en un tercer espacio, que por la división sexual del trabajo es plenamente vivido por las mujeres, y que se halla entre el espacio percibido y mapeado por los ojos de pájaro del poder urbano (masculino) y el espacio imaginado por utopías de mejoras o de control por parte de otros actores políticos externos a este, que igualmente son masculinos (VELASQUEZ-ATEHORTÚA, 2011).

\section{Radicalización de la Democracia Venezolana}

\begin{abstract}
Los demócratas radicales están comprometidos con una participación más amplia en las decisiones públicas. Los ciudadanos deben tener roles más directos en las decisiones públicas, o por lo menos involucrarse más profundamente en las cuestiones políticas substantivas, teniendo la garantía de que sus preocupaciones y opiniones serán efectivamente escuchadas y atendidas por parte de los administradores públicos. En segundo lugar, los demócratas radicales enfatizan la deliberación. En vez de una política de poder e intereses, están a favor de una democracia más deliberativa, en la cual los ciudadanos aborden los problemas públicos por medio de un pensamiento en conjunto sobre la mejor manera de resolverlos - y, como dijo Jürgen Habermas, en la cual "ninguna fuerza está en acción, excepto la fuerza del mejor argumento" (COHEN y FUNG, 2004, p. 23 - 24).
\end{abstract}

La victoria de Hugo Rafael Chávez Frías en el sufragio presidencial de 1998 rompió el patrón de gobiernos neoliberales en América Latina, que caracterizaba a todas las democracias del continente hasta entonces. El eslogan principal de Chávez durante la campaña electoral fue ¡Constituyente Ya!, y la reforma constitucional fue el primer paso en su estrategia para cambiar el sistema político y acabar con los vicios del pasado. Luego un creciente número de naciones vieron alternancias de gobierno hacia la izquierda política, como en Brasil con Luiz Inacio Lula da Silva, electo presidente en el año 2002, Evo Morales Ayma en Bolivia desde 2005, y el Ecuador de Rafael Correa Delgado desde el 2006.

La noción del Socialismo del Siglo 21 emergió paulatinamente ya en los años 1990s, como un reflejo de la desilusión con el neo-liberalismo en Latinoamérica. Lo novedoso del concepto se expresa no simplemente en términos de diferentes contextos históricos y geográficos- sino también en el rechazo al modelo socialista soviético, al considerarse sus rasgos dictatoriales e ineficiente funcionamiento económico. Generalmente hablando, el Socialismo del Siglo 21 promueve un fortalecimiento del rol del Estado en regulación económica y como autoridad dentro de una estructura política democrática. Se determina asimismo por su ambición de una distribución más eficiente y equitativa de los recursos económicos. Se anima la participación a los actores tradicionalmente excluidos, tanto en lo político como en la producción económica (LALANDER, 2011).

La Constitución venezolana bolivariana de 1999 ha sido clasificada como una de las más progresistas del mundo (p.ej. WIPERT, 2007) y estableció la plataforma legal de Hugo Chávez en su ambición de transformar el país. La constitución instituyó la base de un modelo de democracia participativa, en comparación con el sistema representativo y hegemonizado por los dos partidos políticos tradicionales desde la democratización en 1958. La Constitución de 1999 en seguida fue acompañada por legislación secundaria en los temas de participación política y la división político territorial de responsabilidades y autoridades, así como una reforma constitucional parcial en 2009.

El impacto socio-cultural del proceso constituyente fue significativo; Chávez puso la Constitución en la agenda y promovió su accesibilidad para 'ciudadanos ordinarios' como algo que realmente les concernía a ellos. Eso contribuyó a un despertar de interés político entre sectores previamente excluidos y a-políticos (LALANDER, 2011).

Respecto al tema central del artículo, con la Constitución Bolivariana de 1999 asimismo hubo una profundización de los derechos de las mujeres y una ambición hacia una sociedad más igualitaria, como en los artículos 21, 23, 76 y 88. Se manifestó esta tendencia en la manera de formular el texto constitucional, es decir se evitó el lenguaje sexista (GARCÍA y VALDIVIESO, 2009). La socióloga Carmen Teresa García y la politóloga Magdalena Valdivieso han estudiado las relaciones entre el proceso bolivariano y los avances de las mujeres venezolanas. En un artículo de 2009 concluyen lo siguiente:

Podemos decir, sin lugar a dudas, que hay una mayor presencia de las mujeres en el espacio público político acentuada 
inéditamente, esta sola presencia -sin considerar los contenidos de sus accionessignifica un impacto importante, lo que algunas autoras llaman la 'política de presencia' que es válida para todos los grupos excluidos. Estas presencias tendrán efecto en la modificación de los horizontes referenciales de las sociedades y en la ampliación de los contenidos simbólicos culturales: las nuevas generaciones se acostumbran a ver a las mujeres en lo público y no sólo en lo privado-doméstico. Rompiendo también de esta forma el monopolio antidemocrático de los varones en estos espacios (...) Se han abierto importantes expectativas de avance para las mujeres en general, debido a que de muchas maneras se están removiendo las antiguas relaciones de poder en el país y el marco constitucional favorece las posibilidades de que se cumpla con los acuerdos internacionales suscritos por el Estado para eliminar toda forma de discriminación en contra de la mujer y para el pleno reconocimiento a sus derechos (GARCÍA y VALDIVIESO, 2009, p. 150 - 151).

En la sección sobre el contexto histórico del artículo se habló de la dimensión socio-económica y la 'feminización de la pobreza'. No obstante, Venezuela se cuenta entre los países que han logrado reducir dramáticamente la pobreza en tiempos recientes. En cuanto a la estrategia para la reducción de la pobreza y la desigualdad social, a largo y corto plazo se presentan cifras positivas. Entre 1990 y 2010 la pobreza se disminuyó en más del 50 \% y según la ONU se confirma que la pobreza por hogares se redujo del 43.9\% en 1998 a un $26.7 \%$ en 2011 (CEPAL, 2011). Durante el mismo período, la pobreza extrema disminuyó de $17.1 \%$ al 7\% (REPÚBLICA BOLIVARIANA DE VENEZUELA, 2012). Adicionalmente, Venezuela está alcanzando las Metas del Milenio de la ONU en cuanto a la reducción de la pobreza y la igualdad de género, en gran parte a través de las Misiones sociales del Estado (MINMUJER, 2010; AVN, 2011). Las políticas estatales de las Misiones pueden interpretarse tanto como progresistas en sus ambiciones de llevar a cabo una más profunda transformación de las estructuras sociales, pero al mismo tiempo tienen su componente asistencialista/paternalista. Hay una alta participación de mujeres en las Misiones, y en general han alcanzado acceso a beneficios sociales (salud, educación etc.) que anteriormente no tenían (GARCÍA y VALDIVIESO,
2009). Asimismo, Venezuela ha ratificado una cantidad de convenios y tratados internacionales en temas de los derechos humanos de las mujeres ${ }^{2}$. La promoción e implementación de estas medidas se definen en el Plan de Igualdad para las Mujeres Juana Ramírez 'La Avanzadora' 2009-2013 (MINMUJER, 2010). Para la filósofa y feminista Alba Carosio, hay vínculos directos entre el socialismo y la lucha de género.

No hay socialismo posible ni democracia posible si las transformaciones no incluyen la eliminación de las estructuras patriarcales. El patriarcado constituye la forma de institucionalización del poder masculino, que hace parecer como natural su predominio en las estructuras sociales. Se considera justificable, por ejemplo, que los puestos clave del poder (político, económico, religioso y militar) se encuentren, exclusiva o mayoritariamente, en manos de varones. Se considera normal que las mujeres cumplan un rol social y familiar que las somete a muchas más horas de trabajo, que incluyen trabajo remunerado, trabajo doméstico y ahora además, trabajo comunitario (CAROSIO, 2007, s/p.).

\section{Los Consejos Comunales y la Democracia Radical}

Los Consejos Comunales en el marco constitucional de la democracia participativa y protagónica, son instancias de participación, articulación e integración entre las diversas organizaciones comunitarias, grupos sociales y los ciudadanos y ciudadanas, que permiten al pueblo organizado ejercer directamente la gestión de las políticas públicas y proyectos orientados a responder a las necesidades y aspiraciones de las comunidades en la construcción de una sociedad de equidad y justicia social (Artículo 2 de la Ley de Consejos Comunales, 2006). ${ }^{3}$

Las transformaciones políticas y legislativas en Venezuela igualmente conllevaron nuevas connotaciones de la democracia. Mientras que la predominante forma liberal democracia se basaba en un sistema político representativo y colocando los derechos individuales como superiores a los colectivos, en la democracia radical las prioridades 
centrales se enfocan los principios de la voluntad colectiva (mayoritaria), colectivismo, cooperativismo, procesos participativos a nivel local e inclusión de los sectores/ciudadanos que previamente estaban excluidos. La constitución actual de Venezuela (así como las de Bolivia y Ecuador) se caracteriza por una ampliación en los temas de derechos, inclusión social y participación, pero similarmente por un presidencialismo acentuado.

Política e institucionalmente hablando, sin embargo, Venezuela aún tiene estructuras representativas con su origen en un modelo liberal de democracia, incluso en sus procesos electorales. No obstante, desde varios años se han desarrollado experimentos con otros modelos democráticos, en Venezuela y otros países del continente, caracterizados por la ambición de animar a la participación popular y la inclusión de los sectores sociales que previamente estaban marginalizados.

Desde 2006 las unidades organizativas principales de democracia participativa local en Venezuela son los Consejos Comunales, una continuidad práctica de los Consejos Locales de Planificación Pública/CLPP que fueron establecidos ya en 2002 (a través del artículo 182 de la Constitución de 1999 y la ley de los CLPP de 2002). Los Consejos de Planificación experimentaron dificultades en su trabajo de prioridades laborales al nivel comunal y frecuentemente fueron cooptados por los alcaldes municipales. El artículo 184 de la Constitución instituyó los mecanismos y la jurisdicción para la participación popular y el auto-gobierno comunal y vecinal, pero los alcaldes así como los gobernadores regionales aún tenían la posibilidad de intervenir y controlar. La Ley de Consejos Comunales de 2006 fue lanzada para corregir este defecto, y dio a los líderes comunales auto-suficiencia en su relación con políticos de otras instituciones locales. Chávez y otros críticos argüían además que las alcaldías y las gobernaciones mostraban ineficiencia $o$ incluso ignorancia hacia necesidades a nivel vecinal y por eso la ley de los Consejos Comunales fue necesaria (LALANDER, 2012).

Los Consejos Comunales están autorizados para administrar y desarrollar proyectos sociales locales y de organizar actividades dentro de su jurisdicción territorial. Hay más que 30.000 Consejos Comunales en Venezuela. Según cifras oficiales de 2010, este año fueron aprobados $\mathrm{y}$ refundados 21.050 Consejos Comunales (GOLDFRANK, 2011). En comparación con los Círculos Bolivarianos - la forma organizativa principal de las bases del Chavismo entre los años 2000 y 2004 - los Consejos Comunales son organizaciones más grandes. Cada consejo está constituido por entre 200 y 400 familias a nivel urbano y se organiza en diferentes comités temáticos. En las zonas rurales a partir de 20 familias pueden organizarse en un Consejo Comunal y en las comunidades indígenas el mínimo es de diez familias.

En cuanto a la participación dentro de los Consejos Comunales, como ya se mencionó en la introducción, la mayoría de los dirigentes (voceros/voceras) son mujeres, lo que agrega una dimensión cualitativa adicional desde el ángulo de los argumentos de democratización-inclusión en el establecimiento y desarrollo de estas organizaciones de base, es decir un empoderamiento de actores que inicialmente estaban excluidos desde los clivajes de género. Como también se puede observar en los barrios populares con las Juntas de Acción Comunal colombianas o las Organizaciones Territoriales de Base bolivianas, los Consejos Comunales en forma creciente han pasado a ser dirigidas por mujeres amas de casa (VARGAS ARENAS, 2007; LALANDEr, 2012). ${ }^{4}$ Frecuentemente se expresa en Venezuela que'“'La Revolución tiene cara de mujer'. Rosa Reyes, ama de casa y vocera principal de un Consejo Comunal en San Agustín, Caracas, confirma esta dimensión de la transformación participativa:

Antes la participación era baja, muy poca, y la mayoría de los que participaban eran los hombres, en lo político y lo social... Ahora la participación es masiva y la mayoría de los que participamos somos las mujeres. ¿Por qué lo digo? Porque en las reuniones de los comités de tierra la mayoría eran mujeres. En las reuniones de los Consejos Comunales, la mayoría; muchas mujeres. Por eso es que decimos que ahora el proceso es más participativo y de las mujeres. Nuestro presidente dice que es un feminista y que la revolución tiene cara de mujer. Las mujeres andamos participando en todos los procesos, en los Consejos Comunales, en los movimientos de mujeres, en todo lo que es la formación en la universidad bolivariana, en la misión cultura, en todo lo que es el movimiento de participación nosotras estamos insertas allí (Reyes, entrevista, Caracas, 9 de junio, 2011).

La politóloga Sujatha Fernandes (2007) arguye que en general las mujeres de los sectores marginalizados se han fortalecido en cuanto a su participación política, más allá de los Consejos Comunales, en otros programas y misiones sociales de Estado. No obstante, Fernandes también destaca que el culto a Chávez 
tiende a ser fuertemente presente entre las mujeres organizadas. Es necesario destacar, sin embargo, que la insistencia en la lucha vecinal sugiere que hay un impacto en la mentalidad de los ciudadanos, en la percepción de las posibilidades de influir en las decisiones políticas a nivel local. Por cierto, la gran mayoría de estos activistas fueron directamente animados para involucrarse en las políticas organizativas locales por el Presidente Chávez. Pero, en este proceso las mujeres han sido más tenaces y consecuentes que los hombres en términos de asumir responsabilidad y protagonismo en la labor sociopolítica vecinal organizada, normalmente sin ningún tipo de remuneración económica.

Es importante acentuar algunos desafíos y/o contrariedades de los Consejos Comunales. Frecuentemente la estructura de los consejos ha presentado una autonomía débil. Igualmente han dependido altamente de fondos económicos del gobierno a nivel nacional, particularmente del Ministerio de Participación y Protección Social y otras instituciones del Estado. Pero, asimismo pueden solicitar apoyo económico de las autoridades políticas locales y regionales. Los consejos deben presentar una propuesta de proyecto para luego posiblemente tener su aprobación y financiamiento (ELLNER, 2009), lo que fácilmente podría contribuir a una acentuación de clientelismo y paternalismo selectivo del Estado. Este es uno de los retos más difíciles de los Consejos Comunales. En muchos casos, las relaciones entre el Estado nacional y los Consejos Comunales se han caracterizado más por sus rasgos de clientelismo y paternalismo que por un fortalecimiento más genuino y autónomo de la participación popular. Hay, igualmente, casos de malversación de fondos y oportunismo por parte de los dirigentes comunales. Existen otros casos, sin embargo, en los cuales sí hay una participación popular más autónoma, democrática y transparente. El mismo Hugo Chávez estaba consciente sobre los riesgos de que los Consejos Comunales se pudieran convertir en espacios sólo para recibir fondos. En 2007 reflexionaba al respecto:

Ahora miren, sólo alguna reflexión sobre el poder comunal, decía hace un rato ¡cuidado!, tengamos cuidado todos de que los Consejos Comunales no se conviertan sólo en un instrumento para recibir recursos financieros, ¡no!, eso no es lo más importante, eso es muy importante, pero no es lo más importante, vamos a demoler en ese espacio los viejos valores capitalistas del individualismo, del egoísmo, los antivalores más bien, las viejas y malas mañas, vamos a impulsar allí la solidaridad. La cooperación, bueno, el mandato de Cristo, hermanos y hermanas, los que somos cristianos como yo: "Amaos los unos a los otros", allí en la comunidad (CHÁVEZ FRÍAS, 2007, p. 76).

En uno de los primeros estudios académicos sobre los Consejos Comunales, el sociólogo Edgardo Lander (2007) concluyó que los desafíos centrales se encontrarán dentro del Estado y en las tensiones entre dinámicas políticas y estructuras institucionales. Por un lado, el Estado es el promotor tras este proceso democrático, pero por otro lado el mismo Estado constituye un freno para la autonomía de las organizaciones populares. El Estado es por consiguiente tanto el acelerador de las políticas de participación popular y el obstáculo potencial para el desarrollo del mismo proceso. El catedrático Steve Ellner, una de las máximas autoridades académicas en cuanto a la historia política venezolana, resalta que las organizaciones comunales deben examinarse en su perspectiva histórica y que las críticas en términos de autonomía-dependencia de los consejos tienden a exagerarse. La combinación de inversiones estatales en programas sociales (misiones etc.) y el apoyo para proyectos ejecutados por los Consejos Comunales ha animado a los venezolanos de sectores populares para que tomen control de sus propias vidas. Asimismo, Ellner subraya que los chavistas de las bases normalmente no son incondicionales (ELLNER, 2009). En cuanto a la posición crítica de muchos integrantes de los Consejos Comunales, debe subrayarse que hay una creciente cantidad de activistas de la oposición política que han entrado las estructuras políticas comunales, es decir, se muestran conformes con este espacio participativo al nivel local. La vocera Rosa Reyes opina que los avances de los Consejos Comunales han cambiado la vida de los vecinos, al mismo tiempo reconoce algunos desafíos en cuanto al proceso participativo:

Lo interesante de este proceso de los Consejos Comunales, lo novedoso es que es la participación de toda la comunidad, independientemente de las aspiraciones políticas. Hay gente que no milita en ningún partido, hay gente que milita en partidos opositores, y la gente nuestra que simpatiza con el proceso revolucionario. Hay gente de la oposición que reconoce -aunque muy poquita - pero que reconoce por lo menos que este es un logro de la revolución (Reyes, entrevista, Caracas, 9 de junio, 2011).

Es importante mencionar que los avances iniciales 
de los Consejos Comunales coincidían con la orientación oficial explícitamente socialista del gobierno a partir del 2005 y la formación del nuevo partido político oficialista: el Partido Socialista Unido de Venezuela/PSUV. El rumbo hacia un sistema político más socialista, fundamentado en la democracia radical y el poder popular, por consiguiente aceleró a partir del 2006.

En diciembre del 2009 la Asamblea Nacional derogó la ley de Consejos Comunales en su totalidad para aprobar una nueva, esta vez con el carácter de Ley Orgánica. La nueva ley introdujo mayores controles dotando los Consejos Comunales de mejores instrumentos para ejercer contraloría social. Para blindarse de los marcos de corrupción que habían caracterizado la adecuación de una cantidad de Consejos Comunales hasta entonces la Asamblea Nacional reformuló el mismo artículo 2 de la ley en los siguientes términos:

Los Consejos Comunales en el marco constitucional de la democracia participativa y protagónica, son instancias de participación, articulación e integración entre los ciudadanos, ciudadanas y las diversas organizaciones comunitarias, movimientos sociales y populares, que permiten al pueblo organizado ejercer el gobierno comunitario y la gestión directa de las políticas públicas y proyectos orientados a responder a las necesidades, potencialidades y aspiraciones de las comunidades, en la construcción del nuevo modelo de sociedad socialista de igualdad, equidad y justicia social (REPÚBLICA BOLIVARIANA DE VENEZUELA, 2009). ${ }^{5}$

El nuevo artículo precisa cuatro aspectos importantes de analizar. En primer lugar resalta la relevancia de los movimientos sociales y populares, en lugar de la imprecisa alusión a 'grupos sociales' que pueden ser tanto organizados como simples grupos aislados de apoyo social. Con esto se refrendaba el poder organizado del pueblo que en general constituye la base social del proceso revolucionario. En segunda instancia el artículo precisa aún más el papel de los movimientos sociales junto con el pueblo organizado para ejercer el gobierno comunitario. Esta formulación le confiere más autonomía a los Consejos Comunales frente a la forma como estaba formulada su labor en el 2006, donde estos estaban más limitados simplemente a ejercer la gestión de las políticas públicas. El tercer aspecto consiste en considerar las potencialidades de las comunidades, que en a la largo del contenido de la ley sienta las bases para estimular el desarrollo endógeno mediante el proyectos socio-productivos. Finalmente el artículo introduce el adjetivo socialista para calificar el carácter de la nueva sociedad en construcción basada como en artículo del 2006 en la igualdad, equidad y justicia social.

Para acentuar el valor estratégico de las organizaciones comunales en la nueva estructura del Estado (del Poder Popular), el vicepresidente de Venezuela, Elías Jaua, clarifica que esta preferencia incluso de invertir más recursos económicos directamente al nivel comunal- se debe a la visión de reimpulsar "a las comunidades organizadas como núcleo de la descentralización bolivariana y del poder popular" (EL NACIONAL, 20 de abril, 2011). ${ }^{6}$ Carlos Yaguas, director de participación popular del estado Vargas, considera que el nacimiento de los Consejos Comunales y el nacimiento de otras estructuras más complejas de organización popular son ilustrativos de la radicalización de la participación política en Venezuela. En sus palabras:

Venezuela está ante un cambio de estructura, una transformación que era armada o era pacífica. Nosotros estamos transitando por lo pacífico. Entendiendo que hay que ser radical. Una organización como los Consejos Comunales, mas allá de ser una asociación cualquiera de vecinos es una asociación de personas con conciencia de que "estamos transformando la sociedad”. Si no es así no estamos hablando de manera radical. Y hay que actuar de manera radical en este aspecto, no irse a los extremos, sino ser radical (Yaguas, entrevista, Macuto, 3 de junio, 2011).

Se debe destacar que el modelo de democracia participativa a través de los Consejos Comunales de hecho constituye el caso más notorio en participación popular e inclusión social en toda América Latina, considerando que casi la tercera parte de la población adulta participa en estos proyectos. Benjamín Goldfrank compara el desarrollo del modelo comunitario en Venezuela con otros proyectos en América Latina, como en Porto Alegre, Brasil, dónde sólo unos porcientos de la población participó (GOLDFRANK, 2011). Este avance democrático es significativo en términos de equidad de género en las nuevas estructuras participativas -no obstante las debilidades y retos de estas organizacionesconsiderando que la gran mayoría de los activistas en los Consejos Comunales indudablemente son mujeres. 


\section{La Expansión de la Democracia Comunal}

Desde 2010, la democracia participativa venezolana ha expandido, y se presentaron modificaciones legales del sistema de descentralización y la participación popular, entre otros a través de una cantidad de nuevas leyes: Ley Orgánica de Las Comunas; (Nueva) Ley Orgánica del Consejo Federal de Gobierno; Ley Orgánica del Poder Popular; Ley Orgánica de Contraloría Social; Ley Orgánica de Planificación Pública y Popular; y la Ley Orgánica del Sistema Económico Comunal.

La Ley de Comunas significa la posibilidad para un grupo de Consejos Comunales para construir una Comuna. Por su parte, las Comunas pueden unirse para formar una Ciudad Comunal, una evolución que más claramente podría desafiar la autoridad de las alcaldías. Por un lado podría crearse confusión acerca de la jurisdicción entre la ciudad comunal y la alcaldía, pero por otro lado una estructura participativa igualmente podría completar a la otra instancia - la autoridad política representativa - y el éxito de tal sistema de poderes paralelos depende de la capacitación y concientización de los actores, tanto los vecinos y voceros de los Consejos Comunales, las Comunas y las Ciudades Comunales como los alcaldes, gobernadores, concejales municipales, y gerentes y funcionarios en otras instituciones del Estado (LALANDER, 2012). Asimismo es oportuno aclarar que debe haber una dinámica entre las nuevas leyes, el esqueleto jurídico (la Constitución de la República) y el Proyecto Nacional Simón Bolívar con sus Lineas Generales del Plan de Desarrollo Económico y Social de la Nación, 2007-2013, que explícitamente es un plan socialista de desarrollo (MINISTERIO DEL PODER POPULAR PARA LA COMUNICACIÓN Y LA INFORMACIÓN, 2009).

En cuanto a los nuevos espacios para la participación popular, valga aclarar un paso importante en la expansión de la democracia comunal participativa que debe proceder la construcción de una Comuna: las Salas de Batalla Social. Éstas son instancias comunales destinadas a agregar los Consejos Comunales en aras de mejorar la capacidad tanto de organización como de gestión local. Suelen coordinar las peticiones conjuntas que varios Consejos Comunales hacen para acceder a inversiones que vayan más allá de las meramente locales en un consejo comunal particular. Por su carácter pro-oficialista también han sido criticadas por ser 'instrumentos del gobierno' (VARGAS, 2010). El concepto Sala de Batalla Social podría sonar estridente, pero emerge del contexto del cual el que presidente Chávez suele partir para adelantar su gestión. Inicialmente, para hacer su compaña política Chávez llamó a la construcción de Unidades de Batalla Electoral/UBE. Luego de llegar a la presidencia el siguiente paso consistió en convertir estas en Unidades de Batalla Endógenas en las áreas rurales, o en Patrullas de sectoriales en las áreas urbanas. Estas tuvieron la función de reunir las fuerzas locales que habían luchado por alcanzar la victoria electoral y dedicarse a levantar los diagnósticos de necesidades locales. Luego estas mismas unidades se verían abocadas a coordinar la lucha para enlazar los planes de desarrollo de las misiones de la presidencia con la acción concertada de los Consejos Comunales en Salas de Batalla Social.

La evolución del concepto refleja las diferentes etapas que pueden caracterizar la evolución de la participación en la democracia venezolana en la era Chávez. Una de las primeras veces en las que Chávez hizo alusión al concepto fue en el 2008, durante la conmemoración del 13 de abril en un programa de Aló Presidente en la Bombilla, un sector de Petare en el área metropolitana de Caracas. Chávez se refirió al intento de golpe de Estado del que fue víctima el 11 de abril del 2002 y que los habitantes de los barrios populares resistieron con su masiva movilización dos días más tarde. En honor a la rebelión popular esa fecha anunció el lanzamiento de la Misión Presidencial 13 de Abril, M13A, para enaltecer el papel protagónico que jugaron los habitantes de los barrios populares -con alta presencia de amas de casapara defender la democracia venezolana. Chávez precisaba lo siguiente:

Bueno, y un poco para recordarnos que todos los días es 13 de abril, para nosotros todos los días es 13 de abril. Es decir, días de batalla, días de rebelión, contra lo que no sirve... (Aplausos). De rebeldía creadora, transformadora, una revolución es todos los días, y el 13 de abril ocurrió en Venezuela una gran revolución popular, antiimperialista, anti-oligárquica, anti-burguesa que abrió el camino a la nueva era de la revolución socialista, la Revolución Bolivariana (CHÁVEZ FRÍAS, 2008a).

Con estas precisiones ideológicas Chávez explicaba que el objetivo de la misión era por un lado elevar el nivel de vida en los barrios populares para derrotar la pobreza y la miseria y a la vez impulsar el poder popular radicando los valores necesarios 'para ir formando las comunas socialistas' sobre el territorio nacional. La vía para alcanzar este objetivo consistía en que los Consejos Comunales deberían irse 
agregando en barrios comunales y en comunas que entonces el Ministerio de Participación Social y la Fundación para la promoción y el desarrollo del poder comunal (Fundacomunal), habían empezado a impulsar como Salas de Batalla Social (CHÁVEZ FRÍAS, 2008b). Esta doble gestión contra la pobreza y la marginalidad y por la radicación de una nueva ideología política era entonces vista como la fórmula indispensable para erradicar la pobreza y radicalizar la democracia. Previo a estos anuncios el gobierno de Chávez había adelantado una serie de reformas que habían sentado las bases de un espectacular ascenso de la participación de las mujeres en la construcción y ejercicio del Poder Popular mediante el crecimiento aluvial de los Consejos Comunales, como se describió anteriormente.

José Daniel Pérez es un joven vocero principal de la Sala de Batalla Social Unidos por un Nuevo Amanecer Socialista y miembro del Consejo Comunal Esperanza Unida de la Parroquia Caucagüita de Brisas de Turumo en el municipio Sucre, Caracas. Su Sala de Batalla se fundó en el 2008 cuando inicialmente estaba integrada por 42 Consejos Comunales. Por su tamaño, en el 2009 fue sectorizada y del eje del barrio central emergió la Sala, integrada por 19 Consejos Comunales de igual número de sectores. En palabras de Pérez:

Antes éramos solo Consejos Comunales. Y cada quien estaba por su lado. Pero ahora son Salas de Batalla Social y vamos a agruparnos todos. Y esa Sala de Batalla Social es como un espacio, porque no tiene ley que lo respalde. Es simplemente una organización que nace de nosotros mismos como Poder Popular. Alli se abre un espacio de debate para discutir todos los temas de interés y las problemáticas que podamos asumir unidos. Porque la Sala de Batalla Social es la unión de los Consejos Comunales, es como un ensayo para lo que va a ser la conformación de Las Comunas (Pérez, entrevista, Caracas, 12 de julio, 2011).

Cuando nacieron las Salas de Batalla Social no existía la Ley de Comunas - esta fue adoptada por la Asamblea Nacional en diciembre del 2010. Luego, con el apoyo de la nueva ley las Salas de Batalla tuvieron una función más clara de ser una forma organizativa embrionaria de las futuras Comunas. En julio de 2011, ya la Sala de Batalla de Pérez que se mencionó anteriormente había sido preparada como Comuna en Construcción. La construcción de una Comuna puede entenderse como un proceso de sumar a las organizaciones y colectivos activos a nivel local. El proceso involucraba a los vecinos, líderes comunitarios, comerciantes, transportistas, iglesias, los clubes deportivos, los frentes sociales, las Madres del Barrio, los Comités de Tierras Urbanas (CTU), y en general a toda la comunidad que compartiendo unas necesidades comunes se comprometen a trabajar por un bien común. Consiguientemente, en la Sala de Batalla Social confluyen una multitud de intereses sociales, culturales, políticos y económicos, no sólo 'socialistas', y existen sólo por un tiempo limitado ya que es un espacio de preparación para posiblemente desarrollarse en una Comuna.

\section{De la Exclusión al Protagonismo}

Con la revolución bolivariana la mujer venezolana ha logrado ocupar espacios a los que nunca antes había tenido oportunidad de llegar. Hoy vemos a una mujer que ya se ha librado, anda en la calle y tiene protagonismo hoy más que nunca en todos los espacios. Podemos verla en espacios y cargos públicos, dirigiendo los Consejos Comunales, trabajando, estudiando e incluso hasta piloteando aviones (Nancy Pérez, Ministra del Poder Popular para la Mujer, citada en AVN, 2011).

Además del liderazgo tanto en la movilización social como en la postulación de los diagnósticos que abordan las mujeres para 'formalizar' el desarrollo de sus vecindades y ciudades, las mujeres igualmente contribuyen esencialmente al buen desenvolvimiento de dos formas de planificación de la ciudad/vecindad 'formal'. La primera es la planificación denominada como insurgente y la segunda es la planificación comunicativa de corte cohesional, puesta en ejecución bajo la rúbrica del urbanismo social.

Sin lugar a dudas, las mujeres juegan el papel central en las prácticas de ciudadanía insurgente que habían llevado a la radicalización de la democracia venezolana. Libia Mata, promotora de participación en el Estado Vargas, señala que estas tendencias son visibles con la participación de las mujeres en los Consejos Comunales, que superaba entonces el 90 por ciento:

Uno hace las visitas a las asambleas de los Consejos Comunales, y la mayoría de los voceros son mujeres. Y eso lo consigues tú en las mesas técnicas de energía y en las mesas técnicas de agua. Cuando se conformaron los Comités de Tierras Urbanas, estamos hablando del año 2002, cuando se creó el decreto 1.666, nosotros estábamos trabajando promoviendo su 
organización. Y la mayoría han sido mujeres. O sea, desde que llegó Chávez, la mujer realmente se ha visibilizado. Y ha estado a la vanguardia de los procesos orgánicos y de las luchas en cada una de las comunidades. Y eso lo consigue uno a nivel nacional, no solamente a nivel del Estado Vargas (Mata, entrevista, Macuto, 3 de junio, 2011).

Por lo tanto, Mata enfatiza que el incremento de la participación femenina en la política parece haberse solidificado durante la era Chávez a la luz de dos desarrollos territoriales que tomaron inicio con decreto 1.666 del 2002 y la creación de los Comités de Tierras Urbanas. Con el decreto virtualmente terminaron los fenómenos planificados de invasión de tierras urbanas debido a que con éste, el gobierno expresó un compromiso por saldar la deuda histórica con los pobladores de los barrios. Desde entonces una proporción grande del trabajo que las mujeres avanzan como gestoras del barrio ha pasado de la informalidad a la formalidad como lideresas de los programas del Estado, inicialmente en los CTU, luego en los Consejos Comunales, y paulatinamente de ambos dentro de las Salas de Batalla Social. Mata señala además que con la gestación de Salas de Batalla Social se ensancharon las mismas tendencias de amplia participación femenina para alcanzar niveles aún mayores tanto de participación y de eficiencia de gestión.

Aquí en Vargas, en el caso de La Esperanza particularmente ahí hay la mayoría yo podría decir que el $99 \%$ de los que participan responsables de Consejos Comunales son mujeres. El 99\%, y son grupos de mujeres que están a la vanguardia justamente conociendo la realidad.Manejan cual es la realidad. Diagnostican cual es la realidad. Planifican, proponen y ejecutan. Esa es la realidad que nosotros tenemos en cuanto a las mujeres y su relación a través de la sala de batalla social. Como el órgano que los integra a todos para buscar solución a las problemáticas mediatas e inmediatas (Mata, entrevista, Macuto, 3 de junio, 2011).

Mata subrayó además que ese mismo perfil se repetía en otras Salas de Batalla Social de Vargas donde la forma de trabajo de las mujeres coincidía con las observaciones de Juan Velásquez- Atehortúa (2011) en otras ciudades latinoamericanas en términos de su liderazgo comunitario.

Para el vocero José Daniel Pérez hay algunos factores que facilitaban una amplia participación de las mujeres en los Consejos Comunales. Paralelamente a estos en los barrios también existían diferentes Frentes de Mujeres, organizaciones como Madres del Barrio, y diferentes iniciativas socio-productivas organizadas por mujeres. En años recientes, la participación de las mujeres venía siendo notable en la Milicia Popular, donde generalmente son mayoría.Resaltaba que ésta amplia participación femenina también se reflejaba además en la dirección de misiones y ministerios. Según Pérez, las razones de la alta participación de las mujeres en Consejos Comunales eran de dos tipos. La primera la sintetizaba de manera seca.

Porque son más responsables. Yo diría que además tienen mayor humanismo. Porque a veces los hombres no pueden estar pendientes de muchas situaciones. En cambio para hablar lo que es el tema comunal se necesita mucho tiempo, dedicación y amor a esto. Porque esto es voluntario, y las mujeres tienen ese toque, que son bien luchadoras y les gusta (Pérez, entrevista, Caracas, 12 de julio, 2011)

Las apreciaciones de Pérez indican que el mantenimiento de los espacios organizativos de los barrios era una lucha adelantada socialmente por las mujeres que a no solamente disponían de más tiempo, sino también eran más responsables, eran más luchadoras y hacían el trabajo con dedicación y con amor. Todas estas son moralidades, que además hacen parte de los deberes que están consagrados en el articulo 14 de la Ley de las Comunas, al lado de otras virtudes como: la disciplina, la participación, la solidaridad, la integración, la ayuda mutua, la corresponsabilidad social, la rendición de cuentas, el manejo transparente, oportuno y eficaz de los recursos. Estas moralidades por ende se refieren a un espectro de emociones y sentimientos que han pasado de la práctica social informal y cotidiana del trabajo de las mujeres en la esfera del cuidado de la vida ha convertirse en 'practicas ley'. Es decir, la Ley de Comunas en sí formalizaba las prácticas emocionales que antes eran informales.

Desde muy temprano, muchas mujeres son presionadas socialmente a cumplir con su papel reproductivo tempranamente. En este papel son presionadas a mantenerse en relaciones de pareja a costo de su propio desarrollo personal. En la medida en que los hijos crecen las mujeres vuelven a recuperar parte de su autonomía desprendiéndose de la matriz 
reproductiva patriarcal para volcarse a reconstruir su vida social con amigas, vecinas, o compañeras de trabajo. La gran cantidad de madres solas, y mujeres cabeza de familia en la dirección de los Consejos Comunales en los barrios populares confirma este desarrollo. Además muchas de ellas en este papel de lideresas locales encuadran en lo que ha sido discutido por Nina Laurie (2011) bajo el concepto de súpermadres. En cuanto al tema de la feminización de la participación democrática en Venezuela, es conveniente citar a la filósofa y feminista socialista Alba Carosio:

\begin{abstract}
Aunque la Revolución Bolivariana muestra entre sus logros la más amplia participación comunitaria de las mujeres (mesas de agua, Consejos Comunales, misiones, etc.), lo cual es de por sí positivo y ha producido -especialmente en las mujeres de los sectores populares, crecimiento personal, autoestima y sentimiento de valía, esta realidad no ha sido apoyada por esquemas de servicios, mecanismos sociales, ni por transformaciones educativas e ideológicas, que vayan modificando las responsabilidades domésticas y familiares, y las cargas consecuentes. En general, las protagonistas de la participación se ven obligadas a convertirse en heroínas del trabajo que cumplen sus múltiples roles sin fallar en ninguno (CAROSIO, 2007, p. 1).
\end{abstract}

El vocero Pérez sustenta que la mayor parte del trabajo de la Sala de Batalla Social lo realizan las mujeres. Su experiencia es que para elegir determinado cargo siempre tiene que proponerse la presencia de una mujer, quienes por ser más responsables, solidarias, dedicadas, amorosas, luchadoras, etc., pueden garantizar resultados exitosos del trabajo. Esto puede sustentar la prevalencia de las redes sociales compuestas mayoritariamente por mujeres para garantizar el buen funcionamiento de la esfera reproductiva en los barrios. La dedicación de las mujeres a la arena publica en Consejos Comunales y Salas de Batalla Social las hacían de estas un espacio mixto en donde confluyen tanto la producción del barrio como la reproducción de la sociedad, lo que coloca a muchas mujeres ante roces y conflictos familiares.

Pero la mayoría de las veces no hay ningún tipo de problemas. $O$ sea, hacen su cronograma de trabajo y buscan como brindar tiempo al trabajo comunal. En cambio el hombre no. El hombre se la tiene que pasar es trabajando. Las mujeres hacen su qué hacer de su casa, hasta el mismo trabajo [asalariado], y además sus estudios. Pero también le brindan apoyo a lo que es el poder popular, al trabajo comunal, a los Consejos Comunales, y a las luchas sociales y eso. Tienen más tiempo de estar trabajando en su casa. Hacen sus quehaceres y al momento de tener que realizar una reunión ellas participan. Se les ven libres de acceso, no están explotadas, ni nada. Simplemente hacen sus quehaceres y aportan. $Y$ en las reuniones hasta se generan condiciones de trabajo interno para ellas dentro de la comunidad (Pérez, entrevista, Caracas, 12 de julio, 2011)

Esto significa -y contribuye a una explicación de la alta participación de las mujeres en los Consejos Comunales - que por su activa presencia en actividades del cuidado de la vida (reproducción) y de la producción de la vivienda en el barrio las mujeres asumen mayores responsabilidades de control sobre todo lo que es el trabajo a nivel local. Al disponer además tiempo para estudios pueden fortalecer las cualidades de la dirección que ejercían en los Consejos Comunales y otros espacios participativos, como las Salas de Batalla Social.

Ciertamente - regresando al aspecto de politización y dependencia económica por parte de las organizaciones de base - en todos los sistemas democráticos siempre existen riesgos de evaluación arbitraria referente a la aprobación financiera desde arriba de proyectos solicitados desde abajo. Por ende, para su potencial perfeccionamiento los Consejos Comunales dependen - no sólo de los esfuerzos de los integrantes de la estructura comunal- sino también de la actitud de gobernantes a nivel local, regional y nacional las iniciativas progresistas democratizadoras de diferentes instancias del Estado. ${ }^{7}$

La amplia participación de las mujeres en los Consejos Comunales, en el grueso de las organizaciones barriales y en la micro-política local de estos ha sentado las bases del Poder popular como un poder político de influencia de las mujeres. Este poder posiciona al barrio en una serie de direcciones. Primero se puede percibir como un espacio de construcción de sujetos políticos paralelo a los lugares de trabajo de la clase obrera industrial o comercial donde las mujeres tienen más influencia en la construcción del poder popular. Segundo: como un bastión de soberanía territorial de la clase popular, más allá que la clase obrera, cuya composición masculina la habita, pero no 
la dirige. Tercero: como el núcleo territorial en donde la Sala de Batalla Social se erige como un escenario de unidad y concertación de luchas para proteger esta soberanía. En cuarto lugar: se puede entender como un espacio de descolonización o un tercer espacio que con la autogestión de las clases populares confronta tanto a la metrópoli colonizadora como a las subalternas elites colonizadas. Y, finalmente: como una arena donde la Sala de Batalla Social a su vez hace el papel de un (cuarto) espacio critico, en parte dirigido por las mujeres, para dar los debates de descolonización y construir un nuevo orden económico y social.

\section{Reflexiones Finales}

El empoderamiento de las mujeres es un proceso progresivo que ya ha mostrado resultados positivos en cuanto a las prácticas de la aportación de las mujeres de clases populares en los Consejos Comunales. En el presente estudio se ha reflexionado sobre la feminización de la democracia participativa, particularmente a partir del año 2006. Por ende nos preguntamos: ¿La revolución tiene rostro de mujer en Venezuela? Indudablemente hay un aumento visible en cuanto a la presencia representativa y participación activa de mujeres en las organizaciones de base popular.

Desde el ángulo de los seguidores del oficialismo y asimismo por una cantidad de investigadores, el proceso venezolano está compuesto por una radicalización de la participación popular y donde los grandes beneficiarios han sido las mayorías más excluidas. El instrumento para abordar esta radicalización ha sido la programación para reducir la pobreza. La maciza participación popular de las mujeres en estos procesos ha contado con el apoyo decidido del gobierno, que a la vez ha empoderado las prácticas de construcción de sociedad que antes eran adelantadas bajo condiciones de informalidad. Con apoyo estatal estas prácticas obtienen reconocimiento, y pueden elevarse al carácter de formales. Por consiguiente, con esta participación protagónica de las mujeres se fue radicalizando la democracia, haciéndola mas igualada.

Sin embargo, las prácticas históricas y socioculturales del machismo y el marianismo siguen existiendo en las estructuras sociales y en la concepción de los venezolanos, pero simultáneamente la autoestima y el respeto de los esfuerzos de las mujeres enérgicas son avances importantes que han iniciado a afectar la cultura patriarcal. Desde las perspectivas conceptuales de democratización como un aumento de la igualdad política, los avances de las venezolanas de sectores populares en importantes posiciones organizativas locales por sí indican un cambio cualitativo desde el ángulo de paridad de género en los procesos de tomas de decisiones a nivel local/vecinal.

Las mujeres han descubierto 'su espacio de voz y poder' en los Consejos Comunales y otras organizaciones de base. Así que los mecanismos participativos tratados en este estudio y el espacio político y reconocimiento socio-cultural-institucional alcanzado por las mujeres innegablemente pueden ilustrar un ejemplo de ciudadanía insurgente, para reconectar a la conceptualización de Holston. Este protagonismo de mujeres como productoras y administradoras en las emergentes estructuras urbanas se aproxima a esta forma de ciudadanía insurgente de predominio femenino. Es sugestivo el papel que empiezan a asumir las mujeres como constructoras no sólo de la ciudad sino de la ciudadanía desde los barrios populares, los cuáles se han convertido en espacios híbridos, cimentados en gran parte por las mujeres. Es un espacio donde, aún sin escapar al orden patriarcal, racista y capitalista de la sociedad, las mujeres reforman y reformulan la validez de todas estas prácticas, a la vez que vienen poniendo en práctica nuevas adaptaciones con el apoyo del gobierno bolivariano. El enfoque analítico en los Consejos Comunales puede dar algunas claves para entender mejor las transformaciones de la democracia, la ciudadanía, la lucha de género y la participación en Venezuela.

1 Los social-demócratas AD (Acción Democrática) y los demócratas cristianos COPEI (Comité de Organización Política Electoral Independiente).

2 Estos instrumentos internacionales son: Convención para la Eliminación de Todas las Formas de Discriminación Contra las Mujeres (1979), Declaración de las Naciones Unidas sobre la Eliminación de la Violencia Contra la Mujer (1993), Convención Interamericana para Prevenir, Sancionar y Erradicar la Violencia Contra las Mujeres (Convención de Belem do Pará, 1994), y la Cuarta Conferencia Internacional sobre la Mujer (BEIJING, 1995).

3 En 2009 esta ley fue parcialmente modificada.

4 También se ha confirmado mediante observaciones y entrevistas en Venezuela (2007-2012) 
por los dos autores.

5 Palabras en cursiva por quienes suscriben para identificar cambios importantes en comparación con la formulación en el texto legislativo anterior.

6 Artículo: 'Consejos Comunales recibieron Bs 147 millones más que las alcaldías'.

$7 \quad$ El destino tanto de los Consejos Comunales y la democracia radical venezolana más ampliamente hablando, también depende del estado de salud del Presidente Hugo Chávez, quien ha sido tratado por cáncer a partir de junio del 2011. En el momento de entregar el presente manuscrito -diciembre de 2012- el Presidente Chávez se encontraba de nuevo en Cuba para recibir tratamiento médico. A partir de la reelección de Chávez en octubre de 2012, según el plan de gobierno nacional se estima una expansión y profundización de la democracia radical y el poder popular. .

\section{Referências}

AVN - Agencia Venezolana de Noticias. Venezuela cumplió meta del milenio en cuanto a igualdad de género. 2011. [Disponible en: http://www.avn.info.ve/node/47151]

CAROSIO, Alba. La Reforma Constitucional y la perspectiva de género como imperativo ético para la transformación social. 2007. [Disponible en: www.aporrea.org/actualidad/a41379.html]

CEPAL. Balance preliminar de las economías de América Latina y el Caribe 2011. CEPAL, 2011 [Disponible en: http://www.eclac.cl/publicaciones/xml/1/45581/2011 881_BPE_Venezuela_29dic11.pdf]

CHÁVEZ FRÍAS, Hugo Rafael. Aló Presidente del 13 de abril de 2008. Caracas, 2008a. [Disponible en: http://www.alopresidente.gob.ve]

CHÁVEZ FRÍAS, Hugo Rafael. Aló Presidente del 24 de agosto de 2008. Caracas, 2008b. [Disponible en: http://www.alopresidente.gob.ve]

CHÁVEZ FRÍAS, Hugo Rafael. Poder Popular: Alma de la Democracia Revolucionaria, Ministerio del Poder Popular para la Comunicación e Información, Caracas, 2007.

COHEN, Joshua; FUNG, Archon. Radical Democracy.
Swiss Journal of Political Science, v. 10, n. 4, p. 23 34, 2004.

CRASKE, Nikki. Women \& Politics in Latin America. Cambridge: Polity Press, 1999.

EL NACIONAL (varios años). [Disponible en: http://www.el-nacional.com]

ELLNER, Steve. A New Model with Rough Edges: Venezuela's Community Councils. NACLA. Report on the Americas, v. 3, p. $11-14,2009$.

FERNANDES, Sujatha. Barrio Women and Popular Politics in Chávez's Venezuela. Latin American Politics \& Society, v. 49, n. 3, p. 97 - 127, 2007.

GARCÍA, Carmen Teresa; VALDIVIESO, Magdalena. Las mujeres venezolanas y el proceso bolivariano. Avances y contradicciones. Revista Venezolana de Economía y Ciencias Sociales, v. 15, n. 1, p. 133 153, 2009.

GOLDFRANK, Benjamin. Los Consejos Comunales: ¿avance o retroceso para la democracia venezolana? Íconos. Revista de Ciencias Sociales, n. 40, p. 41 $55,2011$.

HOLSTON, James. Insurgent Citizenship in an era of Global Urban Peripheries. City \& Society, v. 21, n. 2, p. $245-267,2009$.

LALANDER, Rickard. ¿Descentralización Socialista? Reflexiones sobre democracia radical, participación política y el neo-constitucionalismo del siglo 21 en Bolivia, Ecuador y Venezuela. Revista Politeia, v. 34, n. 47 , p. $55-88,2011$

LALANDER, Rickard. Venezuela 2010-2011: Polarización y radicalización del proyecto socialista. Revista de Ciencia Política, v. 32, n. 1, p. 293 - 313, 2012 .

LANDER, Edgardo. El Estado y las tensiones de la participación popular en Venezuela. Revista OSAL, v. 8, n. 22 , p. $55-75,2007$.

LAURIE, Nina. Gender Water Networks: Femininity and Masculinity in Water Politics in Bolivia. International Journal of Urban and Regional Research, v. 35, n. 1, p. 172 - 188, 2011.

MATA, Libia, facilitadora de participación del estado Vargas, Macuto, 3 de junio, 2011. 
MINISTERIO DEL PODER POPULAR PARA LA COMUNICACIÓN Y LA INFORMACIÓN. Proyecto Nacional Simón Bolívar: Líneas Generales del Plan de Desarrollo Económico y Social de la Nación, 2007-2013. República Bolivariana de Venezuela, Caracas, 2009.

MINMUJER - Ministerio del Poder Popular para la Mujer y la Igualdad de Género. Informe Preliminar Para la Undécima Conferencia Regional sobre la Mujer de América Latina y el Caribe (CEPAL 2010), CEPAL y MINMUJER, Caracas, 2010. [Disponible en: http://www.eclac.cl/mujer/noticias/paginas/6/38906/Ve nezuela.pdf]

NACIONES UNIDAS. La feminización de la pobreza. En: Conferencia Mujer 2000, Nueva York, 2000. [Disponible en: http://www.un.org/spanish/conferences/Beijing/fs1.htm]

PÉREZ, José Daniel, vocero principal de la Sala de Batalla Social Unidos por un Nuevo Amanecer Socialista, Sucre, Caracas, 12 de julio, 2011.

REPÚBLICA BOLIVARIANA DE VENEZUELA. Ley de Comunas, Gaceta Oficial 6.011 del 21 de diciembre de 2010, Caracas, 2010. [Disponible en: http://www.pgr.gob.ve/dmdocuments/2010/6011.pdf]

REPÚBLICA BOLIVARIANA DE VENEZUELA. Ley de los Consejos Comunales. Reglamento orgánico del servicio autónomo Fondo Nacional de los Consejos Comunales, Gaceta Oficial No 5.806 Extraordinario del 10 de abril de 2006, Caracas, 2006.

REPÚBLICA BOLIVARIANA DE VENEZUELA. Ley Orgánica de los Consejos Comunales, Gaceta Oficial 39.335 del 27 de diciembre de 2009, Caracas, 2009.

REPÚBLICA BOLIVARIANA DE VENEZUELA. Venezuela cumplirá con anticipación las Metas del Milenio de la ONU. [Disponible en: http://venezuelaus.org/es/2012/02/06/venezuela-cumplira-con-

anticipacion-las-metas-delmilenio-de-la-onu/print]

REYES, Rosa, vocera principal, Consejo Comunal de San Agustín, Caracas, 9 de junio, 2011.

SOJA, Edward. Postmetropolis - Critical Studies of Cities and Regions. Oxford: Blackwell publishers, 2000 .
VARGAS ARENAS, Araida. Algunas ideas sobre los Consejos Comunales y la Calidad de Vida de las mujeres populares en Venezuela. Revista Venezolana de Estudios de la Mujer. v. 12, n. 29, p. 33 - 48, 2007.

VARGAS, Adolfo. La praxis de Consejos Comunales y la Visión de las Élites sobre la Democracia Participativa. CIES e-Working paper, No 95, CIES, Lisboa, 2010. [Disponible en: http://www.cies.iscte.pt/destaques/documents/CIESWP95_Vargas.pdf]

VELÁSQUEZ-ATEHORTÚA, Juan. El papel del feminismo barrial en La construcción de la ciudad y la ciudadanía - sus movilizaciones, diagnosis y formas de participar en la planificación en Medellín, Cochabamba y Caracas, ponencia, Trienal de Investigación FAU 2011: Ciudad y Sociedad, Universidad Central de Venezuela, Caracas, 2011.

WILPERT, Gregory. Changing Venezuela by Taking Power, Verso, Londres \& Nueva York, 2007.

YAGUAS, Carlos, secretario de participación popular del estado Vargas, Macuto, 3 de junio, 2011.

Recebido em 4 de dezembro de 2012. Aceito em 25 de março de 2013.

Rickard Lalander e Juan Velásquez-Atehortúa 